\title{
God $x$ evolution of the universe (all possibilities)
}

Author: Sócrates Georges Petrakis.

Degree in Physics, Federal University of Minas Gerais (UFMG); State Department of Education (SEE-MG).

\begin{abstract}
In this proposal, arguments are discussed that reinforce the importance of the observation and application of topics, such as the laws of the conservation of energy and of the total quantity of motion, to guide the development of models complementary to the Big Bang theory. By assuming that the universe contains all of existence at every moment and is thus an isolated system, the cosmos may contain a total amount of energy that is conserved throughout its evolution. Based on this idea, an equation is formulated to guide new suggestions. Figure 1 shows the possibilities of the relationship between God and the Universe.
\end{abstract}

Keywords: God, Universe, Conservation.

\section{Introduction}

The most accepted theory regarding the evolution of the universe posits a certain initial state and currently describes the presence of accelerated expansion [1]. However, the structure generating this expansive beginning is still under debate.

When considering such expansion, studies show that in the beginning, everything occupied a minimum volume. Thus, many analyses of this initial state associate it with the extreme densities present in black holes [2].

Although the use of relativity in most cases indicates the presence of an inner singularity [2], there are cases where this singularity may not be present in a class of black holes called regular [3] [4].

The inequivalence of these cases, related to the fact that these structures do not contain all the mass and energy or all the constituents of the universe, may indicate that this comparison is not very effective.

Thus, a proposal that highlights the observation and application of important conservation laws is developed [5]. 


\section{Development}

The universe can be considered to be the maximum region bounded by the presence of mass and energy or of the components of any formation [6]. Because the universe contains all existence at every moment, the total energy and motion are always conserved [7].

For the analysis of this conservation, a synthesis of the energies that may be present in the high and low density phases is proposed.

Because bosons, antimatter, quantization effects and dark matter may have arisen only after the beginning of expansion, the relativistic energy at rest $\left(E_{0}\right)$ [8] is used to represent all the internal, quantum and structural energy interactions (such as atomic interactions) of any mass at rest $\left[E_{0}=-\left(m_{0}\right.\right.$ : mass at rest) . (c: speed of light) $\left.{ }^{2}\right]$, because when masses are dispersed by dissipation, it corresponds (by conservation) to an energy intensity equal to $E_{0}=+m_{0} \cdot c^{2}$, represented by the wave propagation or linear momentum ( $p$ ) energetically related by de Broglie relations [9] or to the increase in kinetic energy.

Gravitational energy ( $\mathrm{Eg})$, which is also considered to be a negative energy (binding energy), is related to the position of mass [10].

Kinetic energy (Ek), which is never negative, is related to the relative motion of mass or energy (such as waves) and thus to existing space [11].

The total energy of the universe $(E T)$ is analyzed from the following perspective:

$$
E T=-E_{0}-E g+E k
$$

with the negative sign and its corresponding values indicating the intensity of contraction and the positive sign and its corresponding values indicating the intensity of expansion.

The universe does not freely expand because over its evolution, attractive or interacting forces always exist.

When an analysis begins in a state of greater concentration without a structure with massive characteristics, there is no gravitational energy or relativistic energy at rest $\left(-E_{0}\right)$, and thus, the total energy $(E T)$ is represented only by the maximum intensity kinetics exclusively from the motion of energy particles or waves $\left(E^{2}=\left(\left(m_{0} \cdot c^{2}\right)^{2}=0\right)+p^{2} c^{2}\right)[12]$. Because this energy is not a binding energy with a high value, it does not correspond to a state of such intense contraction in a space, which tends to not exist. Thus, before expansion begins, considering the analysis of a universe with massive characteristics is proposed to preserve the law of energy conservation. 
In such a state, everything is compacted in a single point [2] [3], totally or infinitely contracted. When considering a massive composition without a spatial presence, the existence of relative motion is not characterized, and this parameter is not preserved [13].

Thus, a massive state of the highest density necessitates the existence of a smallest possible space, which is related to a spatial dimension [length $=2$. (radius) $=2 . r$ ] and a temporal dimension, corresponding to conservation of motion, with centrifugation (inertia) justifying this tendency.

Centrifugation not only avoids but also promotes a singularity [14]. When considering a massive state (which occupies a place) of maximum density, one cannot have a null dimension. With maximum centrifugation (of the whole universe), there can be deformation, planarization and dispersion to a state of real maximum (one-dimensional spatial) contraction [15].

A given length can generate an infinitesimal volume, provided that the width and thickness are as small as possible.

$$
\text { (Volume }=\text { length. } \text { width. thickness) }
$$

Even being as small as possible, a space present in a spatial dimension would split all of existence into two parts or filaments (2 r r).

Minimal space results in minimal relative motion. In a state contracted to the maximum extent, for conservation of the quantity of motion (QT), the existing mass $(m)$ must be maximal, with the concentration dispersed in an extensive and single spatial dimension, making the moment of inertia (I) maximal. With QT proportional to $\mathrm{m}$. r. w, even considering that the angular velocity (w) is almost null, the product is sufficient for conservation. The universe prior to the expansion, concentrated only in a single infinitesimal point without the presence of any space, does not conserve the total quantity of motion.

The total energy of the maximum contraction or concentration (ET maxc) is negative (bonding) with the maximum value of:

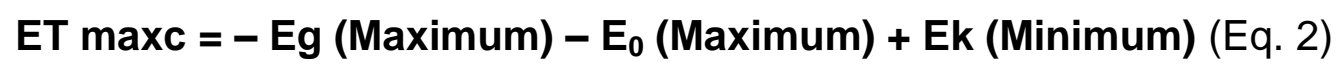

For the maximum gravitational energy, the masses that represent the two parts (with almost zero relative motion) must be equal in quantity.

However, for the existence of any infinitesimal space, the smallest possible part of one of these masses (parts) must be in a state of total dissipation. 
Because of this difference, for maximum contraction before the expansive beginning, one of these structures - separated by the smallest central space reaches a complete state in one spatial dimension, with the other still having an infinitesimal quantity of mass in two spatial dimensions, presenting another smaller space.

Because it is impossible to determine the smallest dissipated mass that generates the smallest space or the largest quantity of mass that existed in the universe in its state of greatest contraction, the following is considered:

$\left(M_{0}+M_{0}\right)=$ maximum total amount of mass in all existence, if spatial presence is not necessary.

$\left(M_{0}+m_{0}\right)=$ total mass if a single and smaller spatial presence is possible, where $\left(M_{0}>m_{0}\right)$ and $\left(M_{0} \cong m_{0}\right)$ (where $(\cong)$ indicates similar or almost equal in quantity).

$E=\left(M_{0}-m_{0}\right) \cdot c^{2}=$ energy that represents the smallest presence of space or the smallest possible amount of mass dissipated in one spatial dimension.

$\left(m_{0}+m_{0}\right)=$ total mass with the two smallest possible spatial presences.

The known universe consists of three-dimensional structures. However, twodimensional or one-dimensional structures are analyzed with the presence of space in two or one dimension where it is considered that there could be real smaller massive particles $\left(\mathrm{M}_{0}-\mathrm{m}_{0}\right)$.

For the instant of maximum concentration, the following presences are proposed:

1) Maximum gravitational energy between the two units or parts, which decreases only after the beginning of expansion.

2) Infinitesimal kinetic energy represented by the relative motion, which is the minimum of the approximation between the parts, in the smallest space existing at the last moment of contraction, which generates the beginning of an expansion.

In the transition from the maximum contraction to the beginning of expansion, the kinetic energy decreases as space approaches the minimum size. Because space cannot cease to exist, the kinetic energy does not become null [16] and intensifies greatly with the beginning of expansion because the gravitational energy and relativistic energy at rest decrease, conserving the total energy [7].

3) Relativistic energy at rest $\left(E_{0}\right)$, which represents the interaction between the two massive halves that exist due to the presence of an infinitesimal central space. 
If all possible masses $\left(m_{0}+m_{0}\right)$ are dissipated, we have an energy equivalent to $\left(m_{0}+m_{0}\right) \cdot c^{2}$, corresponding to the energy at rest [8] (excluding the existing dissipation due to the existence of two infinitesimal spaces).

Therefore, in the state of maximum concentration, when one-half of this quantity of mass is dissipated from the other, the energy at rest equals half of this equivalent.

The other energetic half of this quantity is the binding energy represented by gravity because the infinitesimal kinetic energy is related to the existence of infinitesimal spaces [11].

The presence of an infinitesimal space is caused by the minimum quantity of matter possible $\left(M_{0}-m_{0}\right)$, which is completely dissipated.

Because two infinitesimal spaces are present at maximum contraction, the kinetics can be represented by the energy: $2 .\left(M_{0}-m_{0}\right) \cdot c^{2}$.

Thus, the total energy of a universal state of maximum contraction or concentration (ET maxc) can be represented by:

$$
\begin{aligned}
& E T \operatorname{maxc}=-\left(E g+E_{0}\right)+(E k \cong 0) ; \text { such that } E g=E_{0} ; \\
& \text { ET } \operatorname{maxc}=-\left(2 \cdot E_{0}\right)+(E k \cong 0) \\
& \text { ET } \operatorname{maxc}=-2 \cdot\left(m_{0}+m_{0}\right) \cdot c^{2} / 2+(E k \cong 0) ; \\
& \text { ET } \operatorname{maxc}=-\left(m_{0}+m_{0}\right) \cdot c^{2}+2 \cdot\left(M_{0}-m_{0}\right) \cdot c^{2}
\end{aligned}
$$

There is a positive energy (kinetic) representing an infinitesimal intensity of expansion in a state of maximum, but not complete, contraction.

Considering the current state of accelerated expansion, if all the possible mass that existed at the instant of maximum concentration were completely dissipated in the future, by having its energy summed with that of the infinitesimal spaces that existed, by conservation, we have the following total energy in the future instant of maximum expansion:

$$
\text { ET maxd }=+\left(m_{0}+m_{0}\right) \cdot c^{2}+2 \cdot\left(M_{0}-m_{0}\right) \cdot c^{2}
$$

Because this energy is different in absolute value (or intensity) from the energy of maximum contraction [ET maxc $=-\left(m_{0}+m_{0}\right) \cdot c^{2}+2 .\left(M_{0}-m_{0}\right) . c^{2}$ ], energy is not conserved with complete expansion.

For a maximum expansion (ET maxd) that conserves the total energy, an infinitesimal gravitational energy and relativistic energy at rest, equivalent in value to the kinetic energy in the state of maximum contraction, must exist.

$$
\mathrm{ET} \operatorname{maxd}=+\left[\mathrm{Ek}=\left(\mathrm{m}_{0}+\mathrm{m}_{0}\right) \cdot \mathrm{c}^{2}\right]-\left(\mathrm{Eg} \cong 0+\mathrm{E}_{0} \cong 0\right) ;
$$




$$
\text { ET maxd }=+\left(m_{0}+m_{0}\right) \cdot c^{2}-2 \cdot\left(M_{0}-m_{0}\right) \cdot c^{2} \quad \text { (Eq. 4) }
$$

Thus, the total energy is conserved [7] in absolute value (intensity) by:

$$
\begin{gathered}
\mid \text { ET maxc }|=| \text { ET maxd } \mid \\
\left|-\left(\mathbf{m}_{0}+\mathbf{m}_{0}\right) \cdot c^{2}+\mathbf{2} \cdot\left(\mathbf{M}_{0}-\mathbf{m}_{0}\right) \cdot c^{2}\right|=\left|+\left(\mathbf{m}_{0}+\mathbf{m}_{0}\right) \cdot c^{2}-\mathbf{2} \cdot\left(\mathbf{M}_{0}-\mathbf{m}_{0}\right) \cdot c^{2}\right| \text { (Eq. 5) }
\end{gathered}
$$

One of the total energies results in a negative value representing the maximum contraction and the other in a positive value representing the expansion.

Thus, respecting the law of energy conservation, in its state of maximum expansion, the universe presents the minimum existence of mass.

Having mass and space, even if one or the other is infinitesimal [temperature (T) does not reach absolute zero], energy can never be used to perform the work of volume variation [17] [18] - the universe tends not to expand or dissipate completely but rather to a maximum.

Because in the state of maximum contraction, the energy corresponding to the formation of the two infinitesimal empty spaces is equal to the energy that represents the two infinitesimal masses in the state of maximum expansion, the energy variation between the total mass and total space existing in each of these states is equal, i.e., the total entropy is the same in these extremes.

To be equal, there can be no variation in the total entropy in the volume variation processes of the universe. The increases in entropy required for local thermal equilibrium can be compensated in phases or parts of the universe by constant variations in mass and energy quantity concentrations [19]. Thus, there are expansions followed by contractions of the same intensity.

Boltzmann defined Entropy (S) as a state function:

$\mathrm{S}=\mathrm{k}_{\mathrm{B}}$ (Boltzmann constant). $\operatorname{Ln}(\Omega=$ the state number $)$.

In statistical physics, the state number $(\Omega)=$

$\mathrm{N}$ (the number of possible states). $\mathrm{P}$ (the quantity of movements).

In the state of maximum expansion, the amount of movement and the number of possible states are limited by the energy (space created by longitudinal wave) is one spatial dimension ( $\mathrm{N}$ and $\mathrm{P}$ are Minimal, making $\mathrm{S} \cong 0$ ). 
An outline is given as follows:

Figure 1 (God x Cosmos / Universe):

1) God $=$ Cosmos (all + Nothing $)=$ Eternal $=$ out of the universe in cosmos; 2) God $=$ Universe (all existence $=$ laws of action and reaction); 3 ) God = Universe (The greatest awareness generated by all energy and movement).

Cosmos: $\{$ nothing $\}+\{$ Universe $[$ all existence $=$ Masses (energy) + space (energy) $]\}$

Nothing

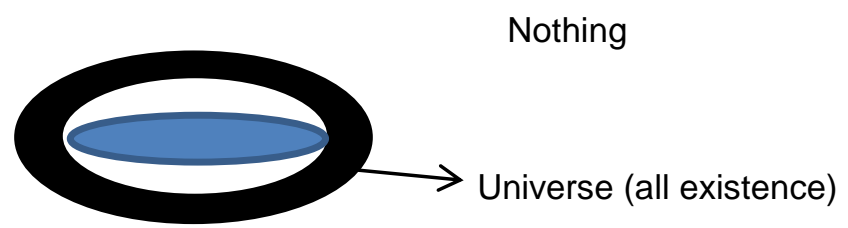

Figure 2 (Universe):

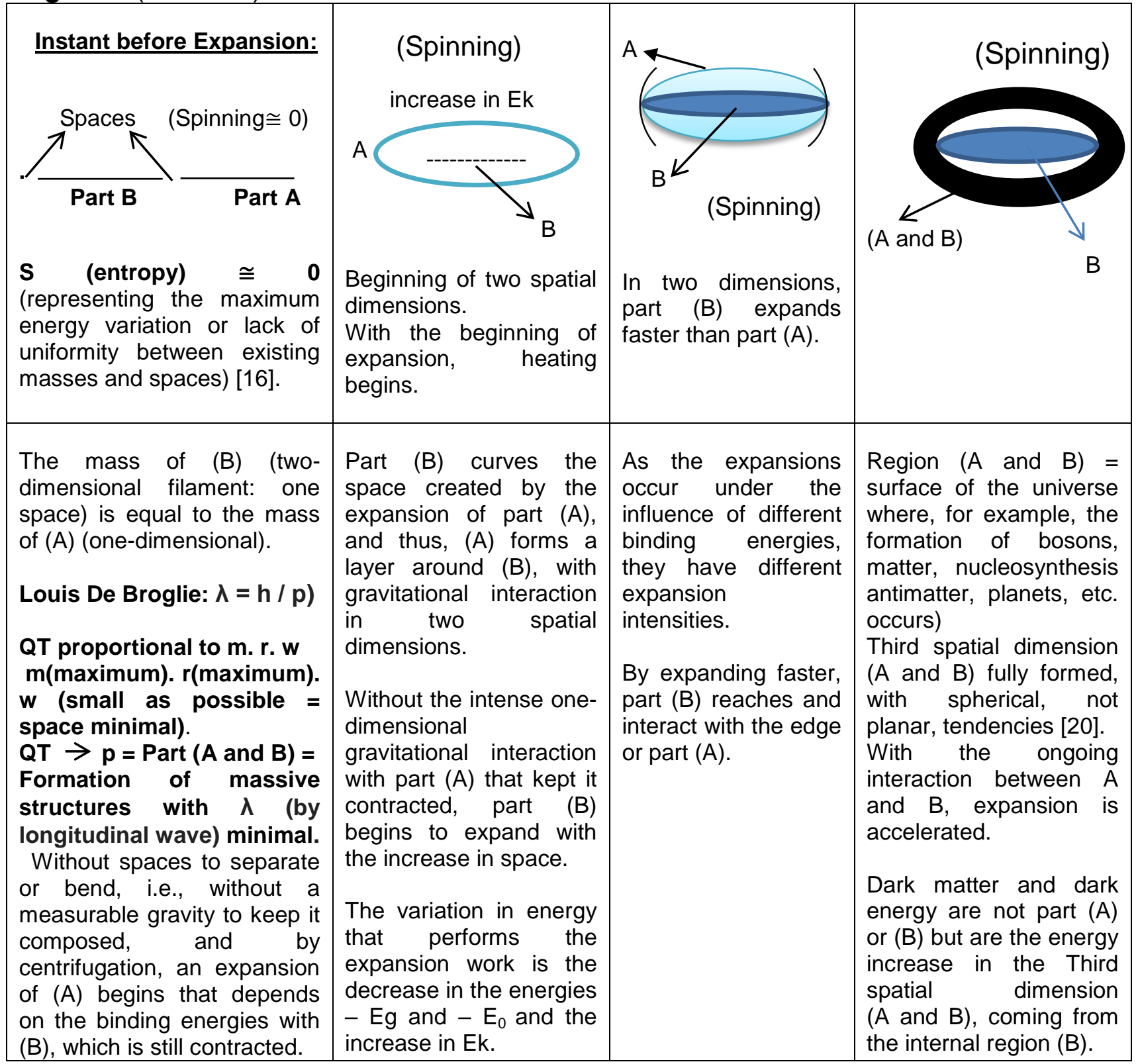


Figure 3 (Universe)

\begin{tabular}{|c|c|c|c|}
\hline (Spinning) & $\begin{array}{l}1 \text { spatial dimension. } \\
\text { Energy (space created } \\
\text { by longitudinal wave). } \\
\text { Gravitational wave } \\
\downarrow \\
\begin{array}{l}(((\cdot))) \\
\left(\mathbf{M}_{0}-\mathbf{m}_{0}\right) . c^{2} \\
\left(\left(\mathbf{M}_{0}-\mathbf{m}_{0}\right) . c^{2}\right. \\
(\text { Spinning } 0))\end{array}\end{array}$ & $\begin{array}{l})_{\text {(Spinning) }} \\
\end{array}$ & (Spinning) \\
\hline $\begin{array}{l}\text { After all of part (B) interacts } \\
\text { with (A), the expansion } \\
\text { starts to decelerate. } \\
\text { With the mass tending to } \\
\text { dissipate, the spinning } \\
\text { decreases. The quantity of } \\
\text { motion (QT) is conserved } \\
\text { by the motion of energy } \\
\text { waves or the creation of } \\
\text { space. } \\
\text { When the maximum } \\
\text { expansion occurs, the } \\
\text { smallest masses possible } \\
\text { emerge: } 2 \text {. }\left(M_{0}-\text { mo } m_{0}\right) \text {, } \\
\text { separated by the maximum } \\
\text { space created by the } \\
\text { largest } E k:\left(m_{0}+m_{0}\right) . c^{2} \text { or } \\
\left(E^{2}=\left(\left(m_{0} \cdot c^{2}\right)^{2}=0\right)+p^{2} c^{2}\right) .\end{array}$ & $\begin{array}{l}\text { With minimal and } \\
\text { nonzero massive } \\
\text { spinning [16] and the } \\
\text { inner space at } \\
\text { maximum, particles } \\
\text { attract one another with } \\
\text { infinitesimal gravity. } \\
\text { Thus, the particles do } \\
\text { not move apart, and } \\
\text { those on the edge move } \\
\text { closer together. } \\
\text { There is no variation in } \\
\text { entropy; dS } 0 \text {, and } \\
\mathrm{S} \cong 0 \text {. Thus, minimal } \\
\text { massive motion, at the } \\
\text { same time as it } \\
\text { becomes null in the } \\
\text { direction of expansion, } \\
\text { begins in the direction of } \\
\text { contraction with the } \\
\text { spinning (increased: } w \text { ). }\end{array}$ & $\begin{array}{l}\text { To come closer, the particles } \\
\text { decrease the one- } \\
\text { dimensional space, i.e., they } \\
\text { absorb energy and thus the } \\
\text { amount of mass increases. } \\
\text { The formation of massive } \\
\text { structures and space in two } \\
\text { spatial dimensions begins. (I) } \\
\text { decreases, (r) decreases, w } \\
\text { increases [21] [22]. } \\
\text { Given that the largest } \\
\text { concentration of energy is } \\
\text { represented by the masses } \\
\text { at the two extremes of the } \\
\text { universe, with the existence } \\
\text { of only an inner space, when } \\
\text { tending toward equilibrium } \\
\text { [23], the quantity of mass at } \\
\text { the edges increases, forming } \\
\text { the second dimension and } \\
\text { subsequently the third } \\
\text { dimension. }\end{array}$ & $\begin{array}{l}\text { Third dimension } \\
\text { with three- } \\
\text { dimensional } \\
\text { space maximally } \\
\text { compacted. } \\
\text { The contraction } \\
\text { continues, and w } \\
\text { (angular velocity) } \\
\text { increases. By } \\
\text { centrifugation and } \\
\text { planarization, } \\
\text { there is a return to } \\
\text { the second spatial } \\
\text { dimension. }\end{array}$ \\
\hline $\begin{array}{l}\downarrow\left(\text { Eg and } E_{0}\right) \\
\uparrow(\text { Spinning }) \\
2^{\text {nd }} \text { spatial dimension }\end{array}$ & $\left.\left(\mathrm{Eg}\right.$ and $\left.\mathrm{E}_{0}\right)\right|_{\text {(Spinning) }} \leftarrow$ & $\begin{aligned} \text { Fart } \mathrm{B} & \\
\text { Future new expansion. } & \\
& \text { Entro } \\
& \text { Entro }\end{aligned}$ & $\begin{array}{l}\text { Part } A \\
\quad(\text { Spinning } \cong 0) \\
\text { py }(S \cong 0) \\
\text { py variation }(d S=0)\end{array}$ \\
\hline $\begin{array}{l}\text { In a massive universe, } \\
\text { when contraction continues } \\
\text { due to the existence of } \\
\text { intense binding energies } \\
\text { that increase and without } \\
\text { the presence of space } \\
\text { (energy) around it instead } \\
\text { of expansion, disintegration } \\
\text { or breakdown of its } \\
\text { components by } \\
\text { centrifugation, deformation } \\
\text { occurs, moving toward } \\
\text { maximum planarization. (r) } \\
\text { And (I) Increases, (w) } \\
\text { Decreases [15]. }\end{array}$ & $\begin{array}{l}\text { Contraction continues } \\
\text { and causes the space to } \\
\text { decrease. The binding } \\
\text { energies increase. } \\
\text { The angular momentum } \\
\text { is preserved, ( } r) \\
\text { increases, (I) increases } \\
\text { and w decreases when } \\
\text { tending to the first } \\
\text { spatial dimension. }\end{array}$ & $\begin{array}{l}\text { The universe returns to the } \\
\text { contraction and the most } \\
\text { separated by a necessary } \\
\text { (which allows conservation } \\
\text { originating from centrifugation } \\
\text { When one of the parts re } \\
\text { thickness (one spatial dim } \\
\text { resumes. }\end{array}$ & $\begin{array}{l}\text { state of maximum } \\
\text { nassive parts are } \\
\text { hore central space } \\
\text { f the total energy) } \\
\text { (inertia). } \\
\text { aches a minimum } \\
\text { ension), expansion }\end{array}$ \\
\hline
\end{tabular}




\section{Conclusion}

All the parts of the universe are energetically related by the interactions between its components $\left(E_{0}\right)$, the positions they occupy $(\mathrm{Eg})$ and the motion (Ek) exerted.

Because it was proposed that the great existing energies are dissipated in massive filaments at maximum contraction or in extensive spaces at maximum expansion with almost null relative massive motions $\left[\left|-\left(m_{0}+m_{0}\right) \cdot c^{2}+\mathbf{2} \cdot\left(\mathbf{M}_{0}-\mathbf{m}_{0}\right) \cdot c^{2}\right|=\left|+\left(m_{0}+m_{0}\right) \cdot c^{2}-\mathbf{2} \cdot\left(\mathbf{M}_{0}-\mathbf{m}_{0}\right) \cdot c^{2}\right|\right]$, in the comparison between these states, the relativistic energy at rest $\left(E_{0}\right)$ was considered, and the typical use of certain relativistic calculations could be discarded [24] [25].

The current existence of the universe in motion already generates variation between the energies with a constant volume variation, always in the direction of thermodynamic equilibrium, which is never completely achieved. The total entropy variation is null [19] [26].

The variation in position, motion, mass quantity, energy and space, which generates work for expansion, is equivalent to the variation in $\mathrm{Eg}_{\mathrm{g}}$ and $\mathrm{E}_{0}$ when converted to Ek, and in contrast, in contraction, the total energy is conserved. In nature, nothing is created or lost; everything is transformed [27].

As any noticeable energy signal is absorbed at the beginning of each cycle, Olbers' paradox can be disregarded [28].

This approach is finalized from the perspective of Occam's razor [29] or the law of parsimony [30], where the best explanation should assume the smallest number of premises. However, new complementary studies are necessary.

\section{References}

[1] Roos, M. Expansion of the Universe - Standard Big Bang Model. In: Engvold, O.; Stabell, R.; Czerny, B.; Lattanzio. J. Astronomy and Astrophysics. 2008.

[2] Hawking, Stephen; Filipe, Bizi and Ellis, G. F. R. The Large Scale Structure of Space-Time. Cambridge: Cambridge University Press. 1973.

[3] Petit, J. P; d'Agostini. G. Cancellation of the central singularity of the Schwarzschild solution with natural mass inversion process Modern. Physics Letters A. 2015; 30, 9.

[4] Neves, J.C. S. Gen.Rel.Grav. 2017; 49,124.

[5] JP Baptista. Revista Brasileira de Ensino de Física. Scielo Brasil. 2006. 
[6] Universe. Webster`s New World College Dictionary. Wiley Publishing, Inc. [S.I.: s.n.]. 2010.

[7] José Plínio Baptista. Revista Brasileira de Ensino de Física. 2006; 28 p.

[8] S. Vieira, A. Barros, I. Araújo e J.C.T. Oliveira. "A comparison among deductions of the equation $E=m c^{2 "}$ (Uma comparação entre as deduções da equação $\mathrm{E}=\mathrm{mc}^{2 "}$ ). Revista Brasileira de Ensino de Física. 2004; V. 26.

[9] Resnick, R.; Eisberg, R. Quantum Physics of Atoms, Molecules, Solids, Nuclei and Particles, 2nd ed. New York: John Wiley \& Sons. 1985.

[10] Lev Davidovich Landau \& Evgeny Mikhailovich Lifshitz. The Classical Theory of Fields. Pergamon Press. 1951.

[11] C., Jain, Mahesh. Textbook of engineering physics: pt. i. [S.I.]: Phi learning. 2009; Chapter 1; 9 p.

[12] JR Forshaw; AG Smith. Dynamics and relativity (Dinâmica e Relatividade), Wiley. 2009; 149, 249.

[13] Cutnell, John D.; Johnson, Kenneth W. Physics 9. ed. (Física 9. ed.) Rio de Janeiro: LTC. 2016; 199 p.

[14] Kerr, R. P. "Gravitational Field of a Spinning Mass as an Example of Algebraically Special Metrics”. Phys. Rev. Let. 1963; 11, $237-238$.

[15] Walker, Jearl. Fundamentos de Física, 10 ed. Rio de Janeiro: LTC. 2016; $305-312$.

[16] Masanes, L., Oppenheim, J. General derivation and quantification of the third Law of thernodynamics (Derivação geral e quantificação da terceira lei da termodinâmica). 2017; Nat Commun 8, 14538.

[17] Tipler, Paul A. Physics 1 (Física 1). Rio de Janeiro: Guanabara Dois. 1978; $448 \mathrm{p}$.

[18] Thomson, W. (Lord Kelvin), On the Dynamical Theory of Heat, with Numerical Results Deduced from Mr. Joule's Equivalent of a thermal Unit, and M. Regnault's Observations on Steam. Transactions of the royal Society of Edinburgh | Vol. 20 |, 1851; | 261- 268 |, | 289 - 298 |.

[19] Kestenbaum, David. Gentle force of entropy bridges disciplines. Science.1998; 279:1849.

[20] Eleonora Di Valentino 1, Alessandro Melchiorri 2* and Joseph Silk 3, 4, 5. Planck evidence for a closed Universe and a possible crisis for cosmology. NATURE ASTRONOMY | VOL 4 |, February 2020; | 196 - 203 |.

[21] Walker, Jearl. Fundamentos de Física. 10 ed. Rio de Janeiro: LTC. 2016; 270-312. 
[22] Ramalho, Nicolau e Toledo. Os Fundamentos da Física - Moderna Plus.

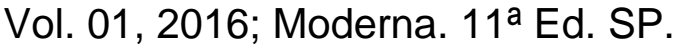

[23] Truesdell, C.A. The Tragicomical History of Thermodynamics, 1980; 1822 1854, Springer, New York, 262.

[24] O'Connor, J.J.; Robertson, E.F; General relativity. Mathematical Physics Index. 1996. Escola de Matemática e Estatística da Universidade de St. Andrews.

[25] Dr. Roberto Belisário e Prof. Luiz Ferraz Netto. What is the special theory of relativity. Science meeting. (O que é a Teoria da Relatividade Especial. Feira de Ciências), 2009.

[26] Van Wylen, G. J. Sonntag, R. E. Borgnakke, C.. Fundamentos da Termodinâmica, 6a ed., Edgard Blücher Ltda. 2003.

[27] Whitaker, Robert D. An Historical Note on the Conservation of Mass. Journal of Chemical Education (52), October 1975; 658 - 659.

[28] Souza, Ronaldo Eustáquio. Introduction to cosmology (Introdução à Cosmologia). São Paulo: Edusp, 2004; 27 p.

[29] Captain Travis Patriquin, US Army. The Principle of the Occam Razor for facts. Military Review (2007).

[30] Ariew, Roger. Ockham's razor: A Historical and Philosophical Analysis of Ockham's Principle of Parsimony. Champaign-Urbana, University of Illinois (1976). 